\title{
Refined discrete and empirical horizontal gradients in VLBI analysis
}

\author{
Daniel Landskron $^{1}$ (D) Johannes Böhm ${ }^{1}$ \\ Received: 10 October 2017 / Accepted: 1 February 2018 / Published online: 20 February 2018 \\ (c) The Author(s) 2018. This article is an open access publication
}

\begin{abstract}
Missing or incorrect consideration of azimuthal asymmetry of troposphere delays is a considerable error source in space geodetic techniques such as Global Navigation Satellite Systems (GNSS) or Very Long Baseline Interferometry (VLBI). So-called horizontal troposphere gradients are generally utilized for modeling such azimuthal variations and are particularly required for observations at low elevation angles. Apart from estimating the gradients within the data analysis, which has become common practice in space geodetic techniques, there is also the possibility to determine the gradients beforehand from different data sources than the actual observations. Using ray-tracing through Numerical Weather Models (NWMs), we determined discrete gradient values referred to as GRAD for VLBI observations, based on the standard gradient model by Chen and Herring (J Geophys Res 102(B9):20489-20502, 1997. https://doi.org/10.1029/97JB01739) and also for new, higher-order gradient models. These gradients are produced on the same data basis as the Vienna Mapping Functions 3 (VMF3) (Landskron and Böhm in J Geod, 2017. https://doi.org/10.1007/s00190-017-1066-2), so they can also be regarded as the VMF3 gradients as they are fully consistent with each other. From VLBI analyses of the Vienna VLBI and Satellite Software (VieVS), it becomes evident that baseline length repeatabilities (BLRs) are improved on average by $5 \%$ when using a priori gradients GRAD instead of estimating the gradients. The reason for this improvement is that the gradient estimation yields poor results for VLBI sessions with a small number of observations, while the GRAD a priori gradients are unaffected from this. We also developed a new empirical gradient model applicable for any time and location on Earth, which is included in the Global Pressure and Temperature 3 (GPT3) model. Although being able to describe only the systematic component of azimuthal asymmetry and no short-term variations at all, even these empirical a priori gradients slightly reduce (improve) the BLRs with respect to the estimation of gradients. In general, this paper addresses that a priori horizontal gradients are actually more important for VLBI analysis than previously assumed, as particularly the discrete model GRAD as well as the empirical model GPT3 are indeed able to refine and improve the results.
\end{abstract}

Keywords VLBI · GNSS · Troposphere · Horizontal gradients

\section{Introduction}

During their passage through the neutral atmosphere, radio waves are delayed and bent as a result of interaction with dry gases and water particles. As there is no chance to directly measure the amount of delay with sufficient accuracy, the delays are usually modeled instead. While the elevation angle-dependent part of the delay is taken into account by the use of mapping functions, the delay also depends significantly on the azimuth of the observation. The ellipsoidal shape of the troposphere as well as the temporally and spatially varying refractivity of the air cause the

Daniel Landskron

daniel.landskron@geo.tuwien.ac.at

1 Technische Universität Wien, Vienna, Austria delays to vary significantly for different observed azimuth angles. In most cases, this effect is considered through horizontal troposphere gradients multiplied with sine and cosine functions, intended to model symmetric variations over the azimuth range. Consideration of these gradients is particularly important for the realization of celestial reference frames (CRFs) (MacMillan and Ma 1997) and terrestrial reference frames (TRFs) (Böhm and Schuh 2007; Mayer et al. 2017). In the analysis of space geodetic techniques such as Global Navigation Satellite Systems (GNSS) and Very Long Baseline Interferometry (VLBI), it has become common practice to estimate gradients on the basis of a very high number of observations. In GNSS, these gradient values are determined and published for instance by the International GNSS Service (IGS), while in VLBI they are important output quantities of analysis software. However, horizontal 
gradients can also be determined from sources other than the actual observations. Ray-tracing through numerical weather models (NWMs) has proven to be well suited for deriving troposphere delays and hence has become the basis for the most accurate mapping functions currently available. In these NWMs, the lower atmosphere is discretized to a temporally varying three-dimensional grid, where the ray-tracing beams then propagate through. Following the Eikonal equation, the ray-tracing beams are delayed and bent, simulating the real travel path as well as possible. As the NWMs are available globally, ray-traced delays can be produced for any point on Earth. The ray-tracing software developed by Hofmeister and Böhm (2017) as part of the Vienna VLBI and Satellite software (VieVS) (Böhm et al. 2017) can not only be used for the derivation of highly accurate mapping functions [see Landskron and Böhm (2017)], but provides the basis for the determination of horizontal troposphere gradients through $2 \mathrm{D}$ ray-tracing at several azimuth angles, too. Depending on the underlying gradient model, the gradients can be realized at all NWM epochs for any site on Earth.

Yet, only minor importance was attached to a priori gradients in VLBI, underlined by the negligible number of existing models and realizations. The Linear Horizontal Gradients (LHG) by Böhm and Schuh (2007) represent the only existing discrete a priori gradient model for VLBI. Calculated directly from NWMs (without ray-tracing), these gradients are provided for all VLBI stations at each NWM epoch, intended for a priori use in VLBI analysis. The term "discrete" in this context means that the gradients are determined discretely for certain locations and times, generally from up-to-date information from ray-tracing through NWMs. In contrast, empirical models rely on experience values from climatology instead. Hereof, two models need to be mentioned: the DAO model from the Data Assimilation Office (MacMillan and Ma 1997) which has been determined by vertical integration over horizontal refractivity gradients, as well as the APG model (Böhm et al. 2013), which first applied the technique of ray-tracing through monthly mean pressure level re-analysis data of the ECMWF. The gradients from these models can then be applied in VLBI analyses as a priori values.

Section 2 first gives a basic understanding of azimuthal asymmetry in troposphere delay modeling. In Sect. 3 the generation of new gradient models is described, whose performance is then assessed in Sect. 4, leading to the conclusions in Sect. 5.

\section{Fundamentals of horizontal gradients}

The modeling of troposphere delays without consideration of azimuthal variations is commonly handled with Eq. (1) [e.g., Nilsson et al. (2013)]:
$\Delta L_{0}(\varepsilon)=\Delta L_{\mathrm{h}}^{z} \cdot m f_{\mathrm{h}}(\varepsilon)+\Delta L_{\mathrm{w}}^{z} \cdot m f_{\mathrm{w}}(\varepsilon)$

The delay is split into a hydrostatic and a wet component, where $\Delta L_{\mathrm{h}}^{z}$ and $\Delta L_{\mathrm{w}}^{z}$ denote the delays in zenith direction and $m f_{\mathrm{h}}(\varepsilon)$ and $m f_{\mathrm{w}}(\varepsilon)$ are the mapping functions accounting for the hydrostatic and the wet part as a function of the elevation angle $\varepsilon$.

In order to model variations in the delays not only depending on the elevation angle but also on the azimuth angle of the observation, a further term must be added to Eq. (1). Gardner (1977) was the first to introduce formulae to compensate for the effect of azimuthal asymmetry. Twenty years later, Chen and Herring (1997) proposed the following formula for the modeling of azimuthal asymmetry:

$$
\begin{aligned}
\Delta L(\alpha, \varepsilon)= & \underbrace{\Delta L_{0}(\varepsilon)}_{\text {isotropic part }} \\
& +\underbrace{m f_{\mathrm{g}}(\varepsilon) \cdot\left[G_{\mathrm{n}} \cdot \cos (\alpha)+G_{\mathrm{e}} \cdot \sin (\alpha)\right]}_{\text {anisotropic part }}
\end{aligned}
$$

which is of common usage in GNSS as well as VLBI analysis down to the present day. The defining variables are the north gradient $G_{\mathrm{n}}$ and the east gradient $G_{\mathrm{e}}$ which determine the variation of the delays with varying azimuth, based on the idea of a tilting of the atmosphere (Herring 1992). The term $m f_{\mathrm{g}}(\varepsilon)$ denotes the gradient mapping function, which models the higher refractivity at smaller elevation angles due to the longer signal path. The representation by Chen and Herring (1997), assuming an exponential decay of the horizontal gradient with increasing height, has prevailed:

$m f_{\mathrm{g}}(\varepsilon)=\frac{1}{\sin (\varepsilon) \cdot \tan (\varepsilon)+C}$

The gradient mapping function coefficient $C$ can be written as:

$C=\frac{3 H}{R_{\mathrm{e}}}$

The scale height $H$ is the height of the neutral atmosphere assuming constant density with height and conservation of the total mass (Nilsson et al. 2013). Assuming a hydrostatic scale height $H_{\mathrm{h}}$ of $6.5 \mathrm{~km}$ and a wet scale height $H_{\mathrm{w}}$ of 1.5 $\mathrm{km}$, Chen and Herring (1997) get values of $C_{\mathrm{h}}=0.0031$ and $C_{\mathrm{w}}=0.0007$ for the gradient mapping function coefficient, $R_{\mathrm{e}}$ being the Earth radius. For modeling total gradients, the factor $C=0.0032$ is recommended (Herring 1992).

Azimuthal asymmetry originates from a number of effects:

- The rotation of the Earth and its resulting centrifugal force not only turn the Earth into an ellipsoid, but act on the atmosphere as well. Consequently, the troposphere is thicker at the equator than at the poles by 
some kilometers. This effect, which is also referred to as the atmospheric bulge, systematically acts on electromagnetic signals traveling through the troposphere, more precisely on the hydrostatic part; the longer a signal's path, the larger its delay. At the equator, the systematic effect is fairly equal for signals from the north and from the south. At the poles, it is equal for all cardinal directions. Given the site of the VLBI station WETTZELL in southern Germany at a latitude of $49^{\circ}$, for instance, signals arriving from the north are less delayed than signals from the south.

- Space geodetic techniques as well as ray-tracing through NWMs usually refer to the reference ellipsoid. However, the real shape of the Earth is much more complex, being referred to as the geoid. Deflections of the vertical (DOV) are the angles between the plumb line and lines perpendicular to the reference ellipsoid at certain locations. In reverse, these DOV can also be visualized as horizontal gradients. They are particularly distinct at plate boundaries or near major mountain ranges.

- Higher temperatures lead to higher convection which lifts the tropopause upwards, which is why the thickness of the troposphere is generally lower in cold conditions and higher in warm conditions (Geerts and Linacre 1997). As a consequence, the tropopause over the poles is up to 2 $\mathrm{km}$ higher in summer than in winter.

- The refractivity along the signal path, which mainly depends on temperature, pressure, humidity, $\mathrm{CO}_{2}$ composition and density (Jones 1981), is highly variable both temporally and spatially. As a result, signals reaching a station from different cardinal directions experience different delays, which is considered as a random effect.

\section{Development of new horizontal gradients}

This section presents the determination of new north gradients $G_{\mathrm{n}}$ and east gradients $G_{\mathrm{e}}$ for the gradient formula by Chen and Herring (1997) as well as for new, higher-order gradient formulae. The main goals for the new gradients are to outperform existing models in VLBI analysis, as well as to improve the baseline length repeatability (BLR) of VLBI analysis w.r.t. estimating the gradients. The basis for the determination are ray-traced delays from the VieVS raytracer applying the 2D piece-wise linear approach (Hobiger et al. 2008). Unlike 1D ray-tracing, in the 2D approach lateral changes in refractivity are also considered.

\subsection{Determination of discrete horizontal gradients for VLBI}

The bulk of this paper is devoted to the determination of new realizations of discrete horizontal gradients based on the standard gradient formula Eq. (2), referred to as GRAD-1, which are then applied in VLBI analysis as well as in delay comparisons. In addition, two extended gradient formulae including higher-order terms are introduced:

$$
\begin{aligned}
\Delta L(\alpha, \varepsilon)= & \Delta L_{0}(\varepsilon)+m f_{\mathrm{g}}(\varepsilon) \cdot \\
& {\left[G_{\mathrm{n}} \cdot \cos (\alpha)+G_{\mathrm{e}} \cdot \sin (\alpha)\right.} \\
& \left.+G_{\mathrm{n}_{2}} \cdot \cos (2 \alpha)+G_{\mathrm{e}_{2}} \cdot \sin (2 \alpha)\right] \\
\Delta L(\alpha, \varepsilon)= & \Delta L_{0}(\varepsilon)+m f_{\mathrm{g}}(\varepsilon) \cdot \\
& {\left[G_{\mathrm{n}} \cdot \cos (\alpha)+G_{\mathrm{e}} \cdot \sin (\alpha)\right.} \\
& +G_{\mathrm{n}_{2}} \cdot \cos (2 \alpha)+G_{\mathrm{e}_{2}} \cdot \sin (2 \alpha) \\
& \left.+G_{\mathrm{n}_{3}} \cdot \cos (3 \alpha)+G_{\mathrm{e}_{3}} \cdot \sin (3 \alpha)\right]
\end{aligned}
$$

The term $G_{\mathrm{n}} \cdot \cos (\alpha)$ determines the azimuthal asymmetry in north-south direction, whereas $G_{\mathrm{e}} \cdot \sin (\alpha)$ determines the azimuthal asymmetry in east-west direction. Thus, one positive and one negative extremum in the asymmetric delay residuals can be modeled. Due to the simple sinusoidal structure of the model, a shortcoming is that a maximum in any azimuthal direction is always accompanied by a respective minimum of opposite sign in an angular distance of $180^{\circ}$. This describes systematic effects like the atmospheric bulge very well, but random effects such as weather fronts or variable atmosphere heights due to local temperature differences set limits in such a way that the consequent extremum does not have a counterpart in the opposite direction. The higherorder gradient variables are intended to model the azimuthal delay variation more closely. The gradients from the standard gradient formula Eq. (2) are henceforth referred to as GRAD-1, those from Eq. (5) as GRAD-2 and those from Eq. (6) as GRAD-3. The term GRAD is used as an umbrella term for all of them.

The gradients $G_{\mathrm{n}}, G_{\mathrm{e}}, G_{\mathrm{n}_{2}}, G_{\mathrm{e}_{2}}, G_{\mathrm{n}_{3}}$ and $G_{\mathrm{e}_{3}}$ have to be determined in least-squares adjustments. The ray-traced slant delays $\Delta L(\alpha, \varepsilon)$ come from ray-tracing following the specifications listed in Table 1 . The elevation angles were picked in such a way as to cover the whole elevation range, while the number of azimuth angles had to be large enough to ensure a sufficient over-determination for the subsequent least-squares adjustment.

First, for each elevation angle and station the slant delays of all 16 azimuths are averaged in order to simulate azimuthally isotropic signals $\Delta L_{0}(\varepsilon)$. Through subtracting $\Delta L_{0}(\varepsilon)$ from the $\Delta L(\alpha, \varepsilon)$, only the asymmetric parts of the delays $\Delta L_{\text {res }}(\alpha, \varepsilon)$ at each azimuth remain. This changes Eq. (2) to:

$\Delta L_{\mathrm{res}}(\alpha, \varepsilon)=m f_{\mathrm{g}}(\varepsilon) \cdot\left[G_{\mathrm{n}} \cdot \cos (\alpha)+G_{\mathrm{e}} \cdot \sin (\alpha)\right]$

Equations (5) and (6) are altered likewise. As the left side of Eq. (7) is known from ray-tracing, the unknowns $G_{\mathrm{n}}$ and 
Table 1 Properties of the ray-traced delays that were generated using the VieVS ray-tracer from 1999 to 2014

\begin{tabular}{|c|c|}
\hline Parameter & Specification \\
\hline Ray-tracing software & $\begin{array}{l}\text { VieVS ray-tracer (Hofmeister and } \\
\text { Böhm 2017) }\end{array}$ \\
\hline Ray-tracing method & $\begin{array}{l}\text { 2D piece-wise linear (Hobiger } \\
\text { et al. 2008) }\end{array}$ \\
\hline NWM & $\begin{array}{l}\text { ECMWF ERA-Interim pressure } \\
\text { level data + ECMWF operational } \\
\text { data }\end{array}$ \\
\hline $\begin{array}{l}\text { Horizontal resolution of the } \\
\text { NWM }\end{array}$ & $1^{\circ} \times 1^{\circ}$ \\
\hline Vertical coverage & 25 pressure levels \\
\hline Horizontal coverage & 33 VLBI stations \\
\hline Temporal resolution & $\begin{array}{l}\text { 6-hourly at 00:00, 06:00, 12:00 and } \\
\text { 18:00 UTC each day from } 1999 \\
\text { through } 2014 \text { ( }=23,376 \text { epochs) }\end{array}$ \\
\hline $\begin{array}{l}\text { Outgoing elevation angles per } \\
\text { point }\end{array}$ & $\begin{array}{l}7\left(3^{\circ}, 5^{\circ}, 7^{\circ}, 10^{\circ}, 15^{\circ}, 30^{\circ} \text { and }\right. \\
\left.\quad 70^{\circ}\right)\end{array}$ \\
\hline Azimuth angles per point & $16\left(0^{\circ}: 22.5^{\circ}: 337.5^{\circ}\right)$ \\
\hline
\end{tabular}

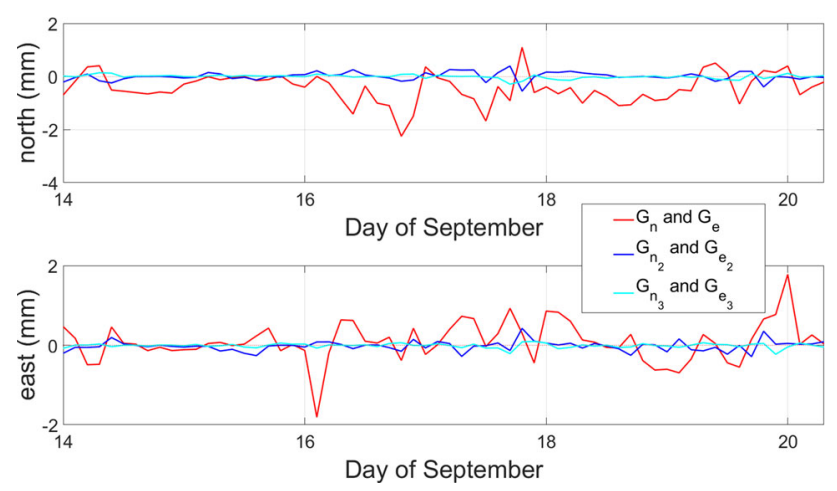

Fig. 1 Comparison of the various gradients for station WESTFORD (Westford, Massachusetts, USA) in mid-September 2011

$G_{\mathrm{e}}$ can be determined through an unweighted least-squares adjustment using partial derivatives. In fact, this is done each for the hydrostatic and the wet part, resulting in gradients $G_{\mathrm{n}_{\mathrm{h}}}, G_{\mathrm{e}_{\mathrm{h}}}, G_{\mathrm{n}_{\mathrm{w}}}$ and $G_{\mathrm{e}_{\mathrm{w}}}$.

Figure 1 indicates that $G_{\mathrm{n}}$ and $G_{\mathrm{e}}$ are considerably larger in size than $G_{\mathrm{n}_{2}}, G_{\mathrm{e}_{2}}$, which in turn are larger than $G_{\mathrm{n}_{3}}$ and $G_{\mathrm{e}_{3}}$.

The capability of GRAD-1, GRAD-2 and GRAD-3 to describe the azimuthal asymmetry can be assessed by determination of the residuals between the modeled delays and the ray-traced delays. Figure 2 shows this exemplarily for VLBI station WESTFORD on September 26, 2011, 18:00 GMT. Averaging the residuals in the slant total delays over all 14 stations and all 15 days of the CONT11 campaign shows a decrease by $69 \%$ when using the standard gradient formula Eq. (2) (GRAD-1), by $78 \%$ when using the second gradient formula Eq. (5) (GRAD-2) and by $81 \%$ when using the third gradient formula Eq. (6) (GRAD-3) compared to nonconsideration of gradients. In other words, two-thirds of the azimuthal asymmetry can be described by the standard gradient formula and even more when using extended gradient formulae. This is a first clear indicator that the extended gradient formulae are indeed capable of describing azimuthal asymmetry more precisely.

\subsection{Determination of an empirical gradient grid}

Apart from the discrete horizontal gradients GRAD, there is also a new empirical gradient grid determined as part of the Global Pressure and Temperature 3 (GPT3) model, providing empirical values for $G_{\mathrm{n}_{\mathrm{h}}}, G_{\mathrm{e}_{\mathrm{h}}}, G_{\mathrm{n}_{\mathrm{w}}}$ and $G_{\mathrm{e}_{\mathrm{w}}}$. Empirical gradient models are needed particularly for observations in the early years of VLBI up to about 1990 (Spicakova et al. 2011), when only few stations were observing a small number of sources, resulting in a non-uniform sky coverage that limits the ability of estimating the gradients in a least-squares adjustment (Heinkelmann and Tesmer 2013). However, empirical gradients may also be important for recent data, for instance for the purpose of deriving terrestrial reference frames (TRFs) from VLBI or for high latitude sites in general where the effect of the atmospheric bulge is most distinct (Böhm et al. 2011).

Currently, only the empirical gradient models APG and DAO are of importance. APG is globally applicable based on a spherical harmonics expansion up to degree and order nine, whereas DAO is only available for a selected list of 174 VLBI stations (as of 2016/05), with new ones being added regularly. Both models provide only total gradients and no separated hydrostatic and wet parts. For VLBI analysis, Böhm et al. (2011) recommend using DAO rather than APG.

For the determination of a new empirical gradient grid, discrete horizontal gradients $G_{\mathrm{n}}$ and $G_{\mathrm{e}}$ (GRAD-1) were calculated first on two global grids following the specifications listed in Table 2. The extended gradient variables GRAD-2 and GRAD-3 are not considered here since their influence is too small for empirical modeling. The next step is to deduce empirical approximations from these discrete gradients, namely mean values of both hydrostatic and wet $G_{\mathrm{n}}$ and $G_{\mathrm{e}}$ for each grid point plus their annual and semiannual amplitudes. The following seasonal fit formula is applied (Lagler et al. 2013; Böhm et al. 2015), providing both a spatial and a temporal variation, exemplified here for the hydrostatic north gradient $G_{\mathrm{n}_{\mathrm{h}}}$ :

$$
\begin{aligned}
G_{\mathrm{n}_{\mathrm{h}}}= & A_{0}+A_{1} \cdot \cos \left(\frac{\text { doy }}{365.25} 2 \pi\right)+B_{1} \cdot \sin \left(\frac{\text { doy }}{365.25} 2 \pi\right) \\
& +A_{2} \cdot \cos \left(\frac{\text { doy }}{365.25} 4 \pi\right)+B_{2} \cdot \sin \left(\frac{\text { doy }}{365.25} 4 \pi\right)
\end{aligned}
$$



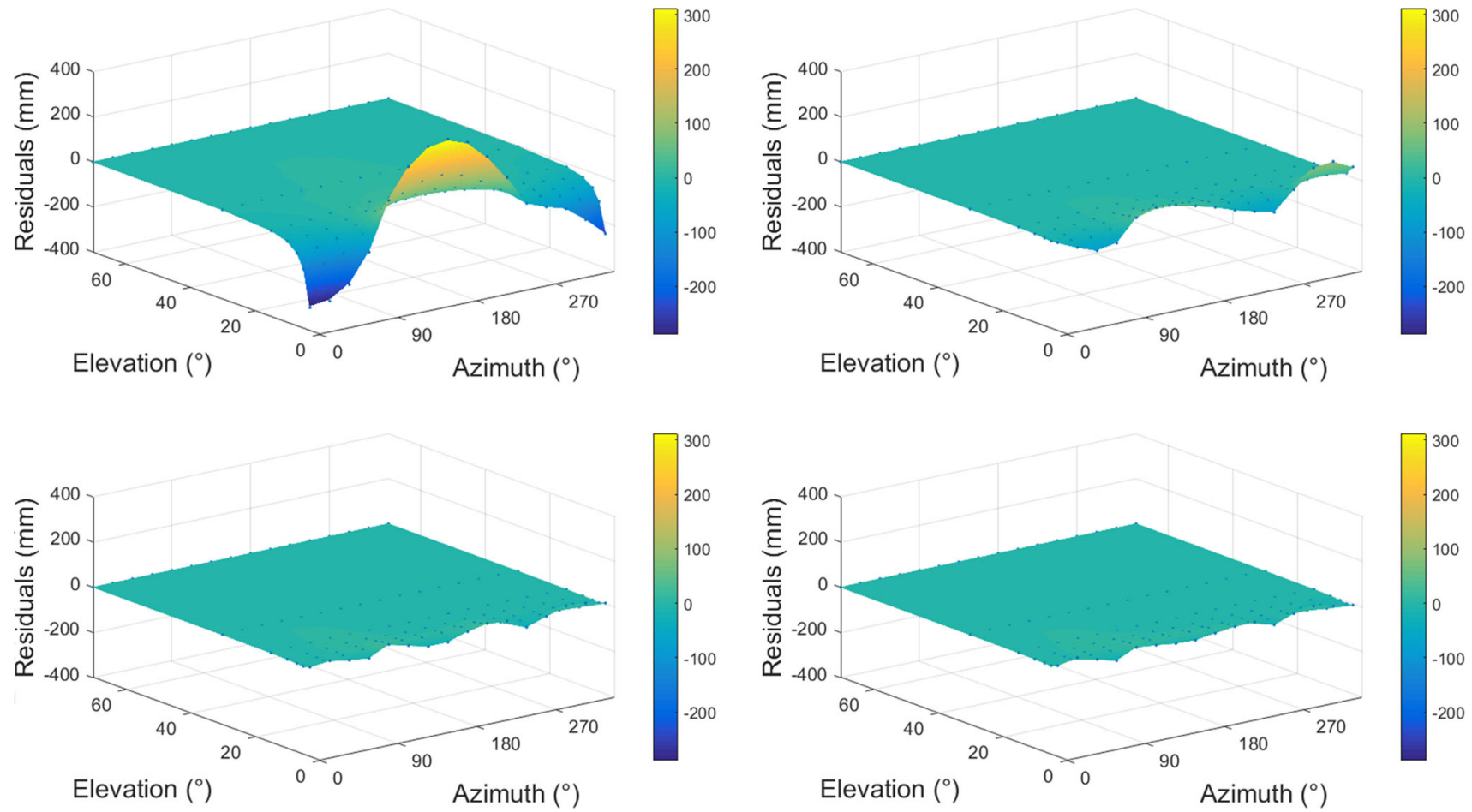

Fig. 2 Comparison of the residuals in slant total delay for station WESTFORD (Westford, Massachusetts, USA) for the epoch September 26, 2011, 18:00 GMT [from Landskron et al. (2015a)]. Top left: residuals after subtraction of a mean over the 16 constantly distributed azimuths without applying any gradient model; the yellow and blue amplitudes mainly show the presence of the atmospheric bulge, the influence of which is highest for low-elevation observations. Top right:

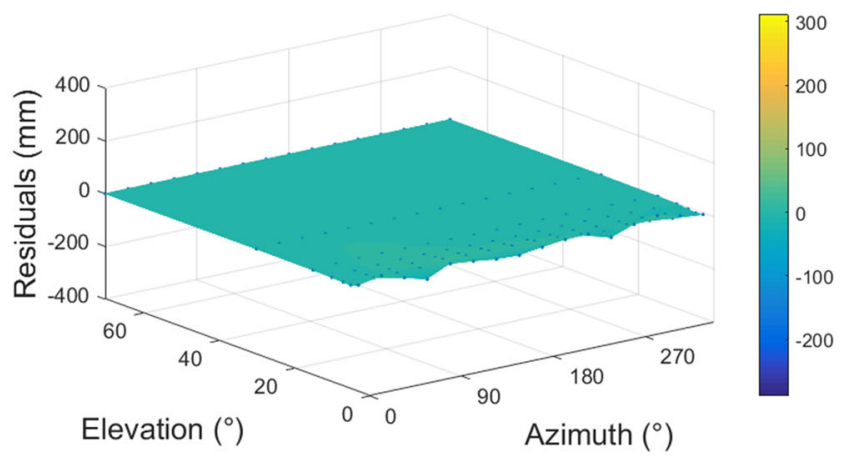

residuals after applying GRAD-1; thus, the bulk of azimuthal asymmetry is explained; however, small amplitudes between the cardinal points remain. Bottom left: applying GRAD-2 further lowers the residuals considerably, also the amplitudes between the cardinal points almost vanish. Bottom right: after applying GRAD-3, the residuals hardly change compared to GRAD-2

Table 2 Properties of the grid-wise ray-traced delays that were generated for the derivation of the empirical gradient grids GPT3 $\left(1^{\circ} \times 1^{\circ}\right)$ and GPT3 $\left(5^{\circ} \times 5^{\circ}\right)$

\begin{tabular}{|c|c|}
\hline Parameter & Specification \\
\hline Ray-tracing software & $\begin{array}{l}\text { VieVS ray-tracer (Hofmeister and Böhm } \\
\text { 2017) }\end{array}$ \\
\hline Ray-tracing method & 2D piece-wise linear (Hobiger et al. 2008) \\
\hline NWM & ECMWF ERA-Interim pressure level data \\
\hline $\begin{array}{l}\text { Horizontal resolution of } \\
\text { the NWM }\end{array}$ & $1^{\circ} \times 1^{\circ}$ \\
\hline Horizontal coverage & $\begin{array}{l}\text { (1) global grid with resolution } 5^{\circ} \times 5^{\circ} \\
\text { (lat: }\left[87.5^{\circ},-87.5^{\circ}\right] \text {, lon: }\left[2.5^{\circ},\right. \\
\left.\left.357.5^{\circ}\right]\right) \text {, resulting in } 2592 \text { grid points } \\
\text { and }(2) \text { global grid with resolution } \\
1^{\circ} \times 1^{\circ} \text { (lat: }\left[89.5^{\circ},-89.5^{\circ}\right], \text { lon: }\left[0.5^{\circ}\right. \\
\left.359.5^{\circ}\right] \text { ) resulting in } 64,800 \text { grid points }\end{array}$ \\
\hline Vertical coverage & 25 pressure levels \\
\hline Temporal resolution & $\begin{array}{l}\text { Mean values for every month from } 2001 \\
\text { through } 2010 \text { (=120 epochs) }\end{array}$ \\
\hline $\begin{array}{l}\text { Outgoing elevation angles } \\
\text { per point }\end{array}$ & $\begin{array}{l}4\left(3.3^{\circ}, 5^{\circ}, 15^{\circ} \text { and } 30^{\circ}\right) \text { for } 5^{\circ} \times 5^{\circ} \text { grid } \\
\text { and } 1 \text { elevation }\left(3^{\circ}\right) \text { for } 1^{\circ} \times 1^{\circ} \text { grid }\end{array}$ \\
\hline Azimuth angles per point & $8\left(0^{\circ}: 45^{\circ}: 315^{\circ}\right)$ \\
\hline
\end{tabular}

where $A_{0}$ represents the mean value, $A_{1}$ and $B_{1}$ the annual amplitudes, $A_{2}$ and $B_{2}$ the semiannual amplitudes of $G_{\mathrm{n}_{\mathrm{h}}}$ and doy the day of year. Again, least-squares adjustments are applied in order to fit $A_{0}, A_{1}, B_{1}, A_{2}$ and $B_{2}$ to the discrete gradients at each point of the grid. Users can eventually determine the actual gradients for the exact time and location of their measurement through bilinear interpolation from the surrounding grid points. These empirical horizontal gradients are part of the new empirical troposphere model Global Pressure and Temperature 3 (GPT3) (Landskron and Böhm 2017), optionally in $1^{\circ} \times 1^{\circ}$ and $5^{\circ} \times 5^{\circ}$ resolution. Figure 3 shows mean values, cosine amplitudes and standard deviation of $G_{\mathrm{n}_{\mathrm{h}}}$, while Fig. 4 involves the same without the cosine amplitudes for $G_{\mathrm{e}_{\mathrm{h}}}, G_{\mathrm{n}_{\mathrm{w}}}$ and $G_{\mathrm{e}_{\mathrm{w}}}$. In the top left plot of Fig. 3, the systematic effect of the atmospheric bulge is predominant. The hydrostatic part generally affects regions outside the tropics, while the wet part is most distinct roughly between $25^{\circ} \mathrm{N}$ and $25^{\circ} \mathrm{S}$ (center left plot of Fig. 4). In the top left plot of Fig. 4, the systematic effect of the deflections of the vertical can be seen, which are very distinct near dominant mountain ranges such as the Andes or at plate boundaries such as around Japan. The wet gradients (center left and bottom left plots of Fig. 4) are mainly affected by trade winds. 

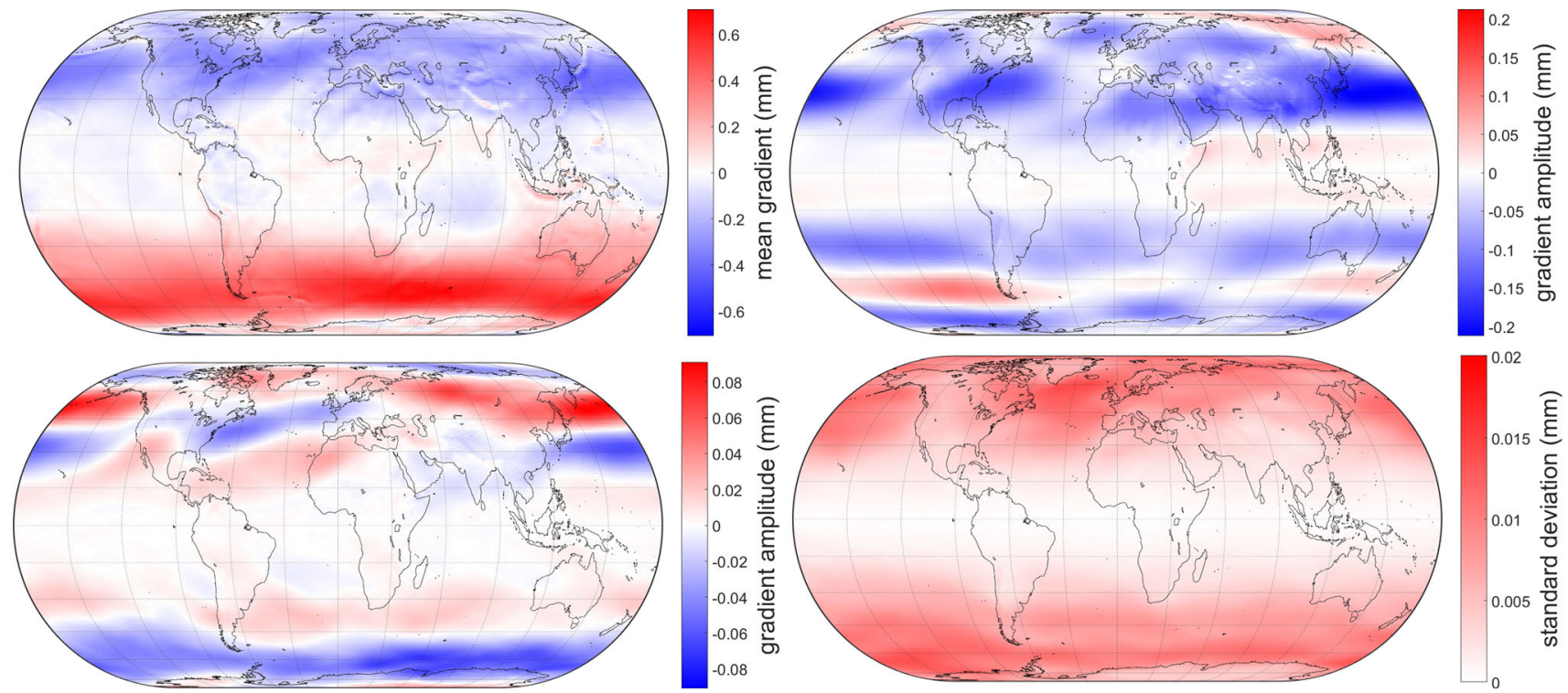

Fig. 3 Mean values $A_{0}$ (top left), annual amplitudes $A_{1}$ (top right), semiannual amplitudes $A_{2}$ (bottom left) and standard deviation of the residuals (bottom right) of the hydrostatic north gradient $G_{\mathrm{n}_{\mathrm{h}}}$ from GPT3. $B_{1}$ and $B_{2}$ are not included, as they are very similar to $A_{1}$ and $A_{2}$
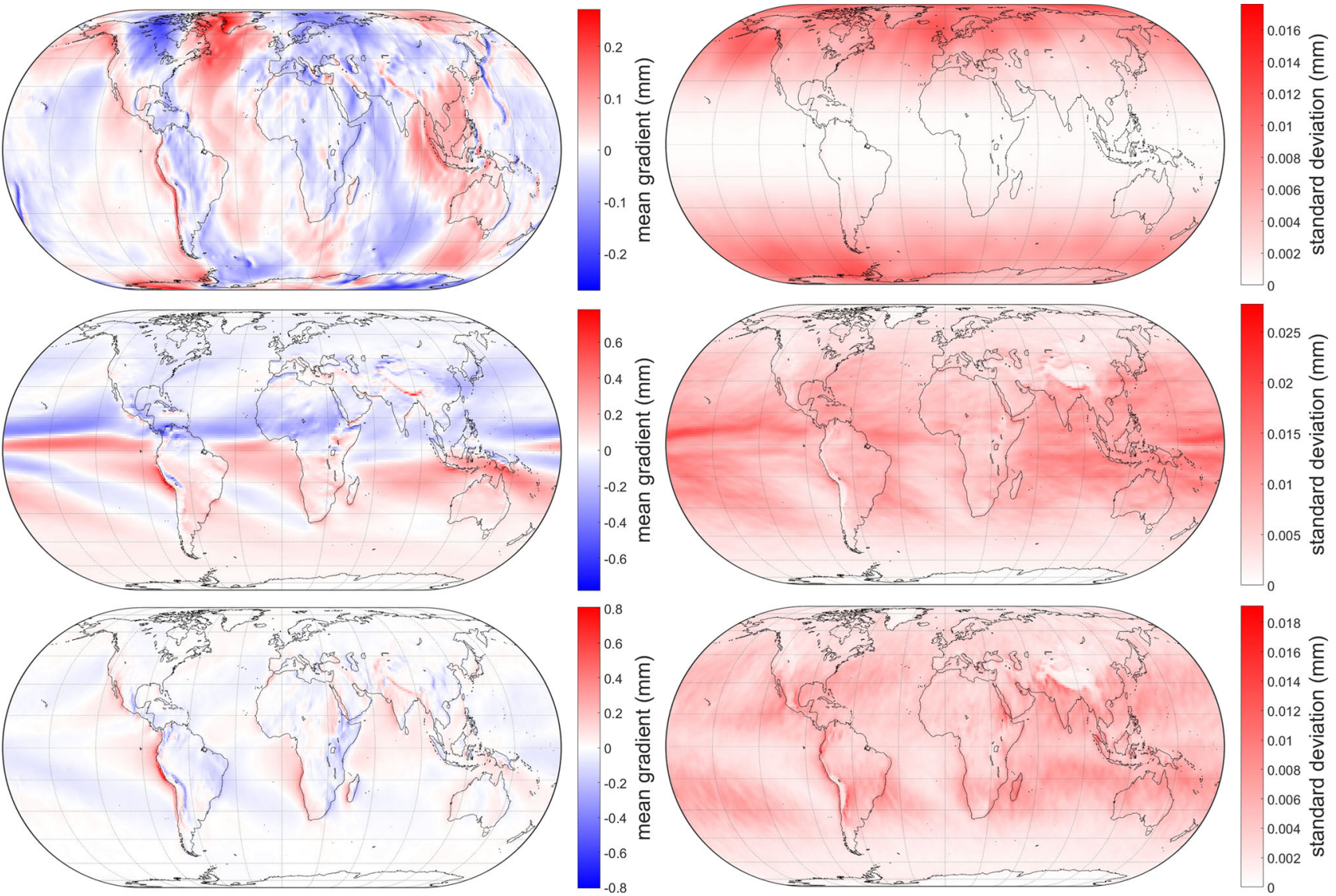

Fig. 4 Mean values $A_{0}$ (left) and standard deviations of the residuals (right) of the hydrostatic east gradient $G_{\mathrm{e}_{\mathrm{h}}}$ (top), wet north gradient lack of additional information, the amplitudes $A_{1}, B_{1}, A_{2}$ and $B_{2}$ are not included here 

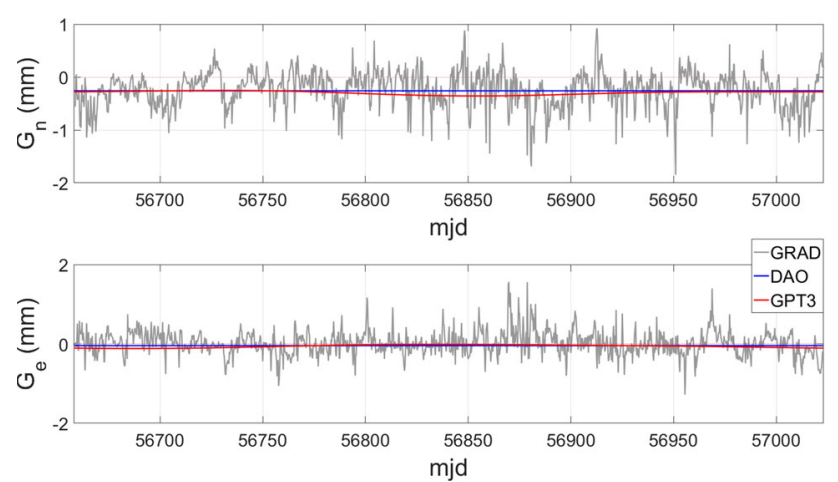

Fig. 5 Comparison between total discrete and empirical north (top) and east (bottom) gradients for station WETTZELL during spring 2014. In case of GPT3, the gradients for a specific site are determined through bilinear interpolation from the four surrounding grid points

Empirical gradients, however, only have the ability to describe a small, apparently insignificant part of the actual, discrete gradients, which is outlined in Fig. 5. Unlike DAO, the GPT3 gradients possess a small time-dependent component, although there is no chance to sufficiently describe the significant random, short-term variations due to weather events dominating the behavior of the discrete gradients.

\section{Comparisons and results}

In order to assess the quality of GRAD and GPT3, several comparisons are undertaken. First, BLRs are determined from VLBI analyses using VieVS, as shown in Sect. 4.1. Nine years of VLBI data including 1338 observation sessions are analyzed for this purpose, where only sessions with at least 3 observing stations were picked, eliminating all intensive sessions. Secondly, the gradients are used to model delays which are then compared to ray-traced delays (Sect. 4.2). The better the gradients approximate the ray-traced delays, the higher their accuracy is assumed to be. These comparisons are done on a global grid with a horizontal resolution of $5^{\circ} \times 5^{\circ}$.

\subsection{Comparison of BLRs}

Baseline length repeatabilities are an appropriate measure to assess the quality of geodetic VLBI products (Böhm and Schuh 2004; Titov 2009). The lower the BLR, the better the performance of a certain model. Table 4 shows the resulting BLRs from VLBI analyses of several models, as averaged over 1338 VLBI sessions from 2006 to 2014 . The ray-traced delays, which serve as the basis for the determination of the GRAD a priori gradients, were computed following the specifications in Table 1. They were then interpolated to the VLBI observation epochs through spline interpolation. The settings for the VLBI analyses are listed in Table 3. The results of
Table 3 Setting for the VLBI analysis using VieVS

\begin{tabular}{|c|c|}
\hline Option & Decision \\
\hline Mapping function & $\begin{array}{l}\text { Vienna Mapping Functions } 1 \\
\text { (VMF1) (Böhm et al. 2006) }\end{array}$ \\
\hline Terrestrial reference frame & VieVS TRF (Böhm et al. 2017) \\
\hline Celestial reference frame & $\begin{array}{l}\text { International Celestial Reference } \\
\text { Frame } 2 \text { (ICRF2) }\end{array}$ \\
\hline Tidal ocean loading & FES2004 (Lyard et al. 2006) \\
\hline $\begin{array}{l}\text { Tidal and non-tidal } \\
\text { atmosphere loading }\end{array}$ & VIENNA (Wijaya et al. 2013) \\
\hline $\begin{array}{l}\text { Estimate } \Delta L_{w}^{z} \text { within the } \\
\text { analysis }\end{array}$ & $\begin{array}{l}\text { Yes; as piece-wise linear offsets } \\
\text { hourly using relative constraints } \\
\text { of } 1.5 \mathrm{~cm}\end{array}$ \\
\hline $\begin{array}{l}\text { Estimate gradients within } \\
\text { the analysis }\end{array}$ & $\begin{array}{l}\text { if desired; as piece-wise linear } \\
\text { offsets 6-hourly using relative } \\
\text { constraints of } 0.5 \mathrm{~mm} \text {, but no } \\
\text { absolute constraints }\end{array}$ \\
\hline
\end{tabular}

Table 4 Mean BLRs (cm) from VLBI analyses for all 1338 sessions from 2006 to 2014. In column (1), only a priori gradients are used, while in column (2) the gradients are additionally estimated in the VLBI analysis using the standard gradient formula

\begin{tabular}{lll}
\hline Gradient model & $(1)$ & $(2)$ \\
\hline (a) No a priori gradients & 1.68 & 1.65 \\
(b) LHG & 1.66 & 1.67 \\
(c) GRAD-1 & 1.58 & 1.66 \\
(d) GRAD-2 & 1.57 & 1.65 \\
(e) GRAD-3 & 1.58 & 1.65 \\
(f) APG & 1.65 & 1.66 \\
(g) DAO & 1.64 & 1.66 \\
(h) GPT3 $\left(5^{\circ} \times 5^{\circ}\right)$ & 1.63 & 1.66 \\
(i) GPT3 $\left(1^{\circ} \times 1^{\circ}\right)$ & 1.63 & 1.66 \\
Ray-traced delays & 1.57 & 1.64 \\
\hline
\end{tabular}

Table 4 are surprising because the estimation ${ }^{1}$ of gradients in the VLBI analysis degrades the resulting BLRs. Best results are achieved when using a priori gradients without estimation of the gradients. GRAD-2 yields the best performance, improving the BLRs of $43 \%$ of the stations by more than 1 $\mathrm{mm}$ while degrading only $5 \%$ of the stations by more than 1 $\mathrm{mm}$ (the complementary $52 \%$ are between -1 and $+1 \mathrm{~mm}$, too small to be referred to as an improvement or degradation). Figure 6 outlines this more closely, assuming no gradients were estimated in the VLBI analysis. Figure 7 shows that the improvement from the a priori gradients is most distinct for shorter baselines. This is most likely because horizontal gradients affect horizontal positions in particular. Since

\footnotetext{
1 The estimation of gradients is done with the standard gradient formula Eq. (2); an estimation using the extended gradient formulae Eqs. (5) and (6) was tested as well, but turned out to not improve the results.
} 


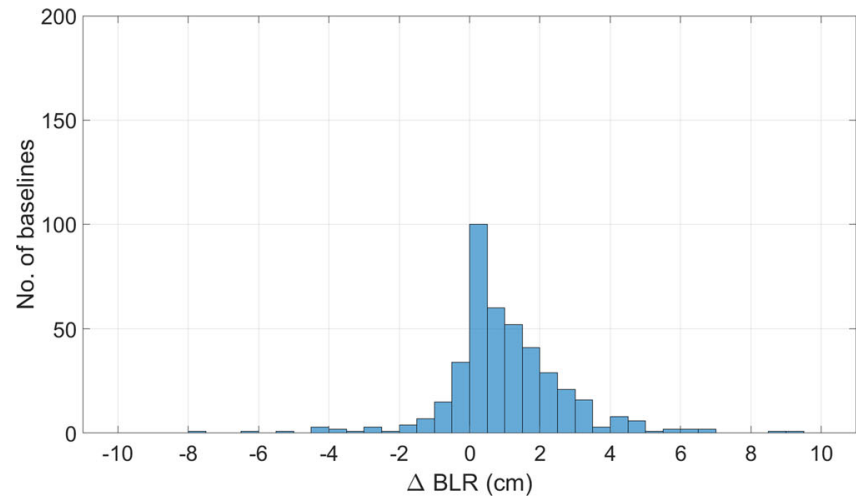

Fig. 6 Difference in BLR (cm) from VLBI analysis for all VLBI sessions from 2006 to 2014 without gradient estimation. Left: using GRAD-2 compared to no a priori gradients; right: using GPT3 $\left(1^{\circ} \times 1^{\circ}\right)$

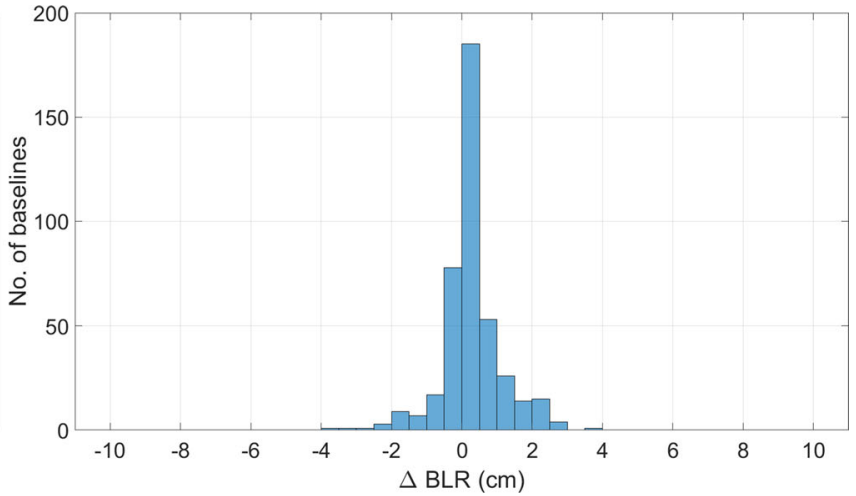

compared to no a priori gradients. The bars in the positive range imply improvement each

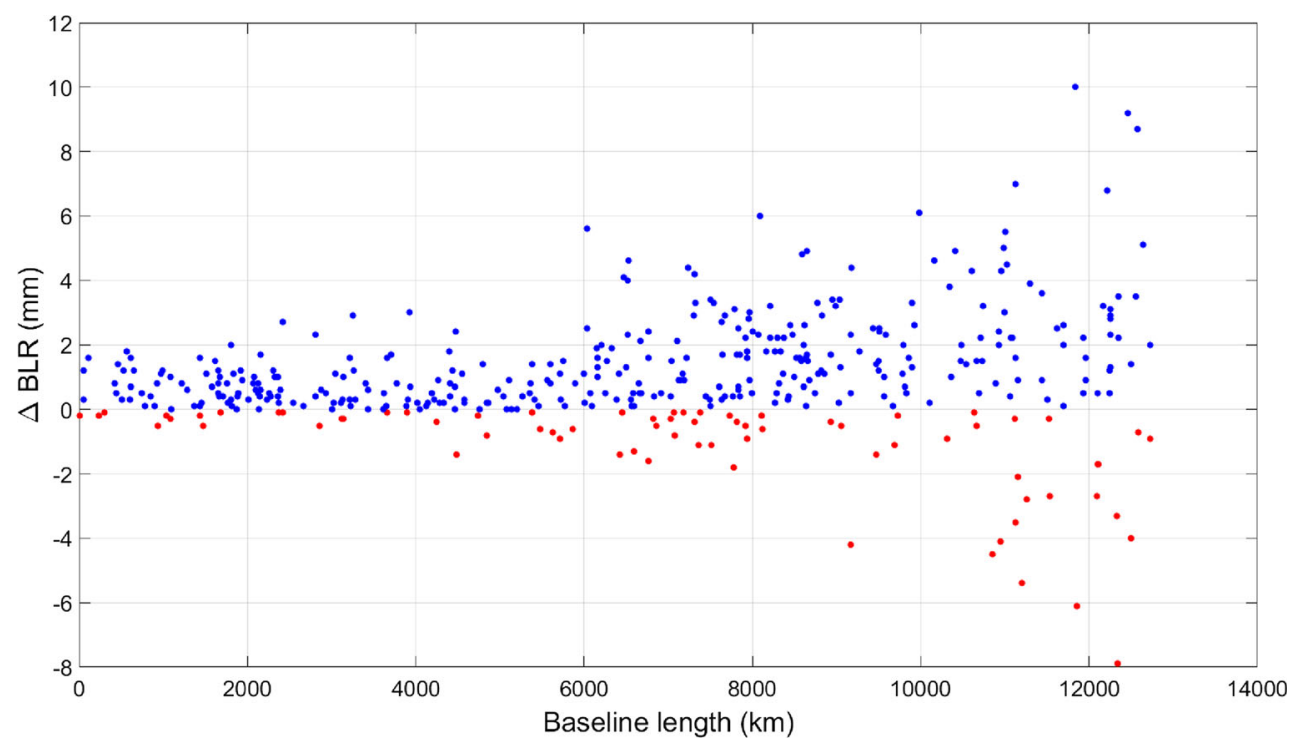

Fig. 7 Difference in BLR (cm) from VLBI analysis for all VLBI sessions from 2006 to 2014 without gradient estimation, using GRAD-2 compared to no a priori gradients. Blue dots indicate improvement

baselines run straight through the Earth, their repeatability is less affected by horizontal position changes with increasing baseline length.

There is a stark contrast to the results from Landskron et al. (2015b), who concluded that estimating gradients yields best results in any case. The essential difference is that Landskron et al. (2015b) analyzed only two weeks of VLBI data, more precisely the CONT11 campaign. Each session of CONT11 consists of a vast number of observed baselines, in fact more than 4000, providing an optimal basis for the gradient estimation. As a consequence, the lowest BLRs are achieved with the estimation. Although the results of Table 4 contain such sessions as well, the vast majority of sessions comprises only a few hundreds of observations. This substantially impairs the quality of the estimated gradients and is finally reflected in moderate BLRs. through using GRAD-2. It can be seen that the improvement is most distinct for shorter baselines

Apparently, a session must have a minimum number of observations in order to get reliable results. To prove this assumption, various tests were made which yielded an appropriate boundary value of 3000 observations per session, below which no gradient estimation shall be done. ${ }^{2}$ VLBI analyses carried out separately for all VLBI sessions containing fewer than 3000 observations and for those containing more than 3000 observations result in Table 5. This unambiguously proves the assumption that gradient estimation using a least-squares adjustment shall only be done for sessions possessing a sufficient number of observations.

\footnotetext{
2 There were several strategies tested for a boundary value regarding total number of observations, mean number of observations per station, number of scans and some more. However, the most general criterion (total number of observations) turned out to be the most appropriate one at the same time. See Landskron (2017) for details.
} 
Table 5 Mean BLRs (cm) from VLBI analyses for all those sessions from 2006 to 2014 that contain fewer than 3000 observations (1129 out of 1338 sessions, columns 2 and 3 ) and more than 3000 observations (209 out of 1338 sessions, columns 4 and 5). In (1), only a priori gradients are used, while in (2) the gradients are additionally estimated in the VLBI analysis

\begin{tabular}{llllll}
\hline Gradient model & \multicolumn{2}{l}{$<3000$ observations } & & \multicolumn{2}{c}{$>3000$ observations } \\
\cline { 2 - 3 } \cline { 5 - 6 } & $(1)$ & $(2)$ & & $(1)$ & $(2)$ \\
\hline (a) No a priori gradients & 2.32 & 2.38 & & 1.08 & 0.97 \\
(b) LHG & 2.32 & 2.36 & & 1.06 & 1.04 \\
(c) GRAD-1 & 2.21 & 2.39 & & 1.00 & 0.99 \\
(d) GRAD-2 & 2.19 & 2.37 & & 1.01 & 0.98 \\
(e) GRAD-3 & 2.19 & 2.38 & & 1.01 & 0.99 \\
(f) APG & 2.25 & 2.39 & & 1.09 & 0.97 \\
(g) DAO & 2.23 & 2.39 & & 1.09 & 0.97 \\
(h) GPT3 $\left(5^{\circ} \times 5^{\circ}\right)$ & 2.23 & 2.39 & & 1.08 & 0.97 \\
(i) GPT3 $\left(1^{\circ} \times 1^{\circ}\right)$ & 2.23 & 2.39 & & 1.07 & 0.97 \\
Ray-traced delays & 2.18 & 2.33 & 1.01 & 0.99 \\
\hline
\end{tabular}

Above 3000 observations per session the gradients shall be estimated, whereas below this boundary it is strongly recommended to not estimate them as the least-squares adjustment will most likely not output well-fitting gradients. This is new as the commonly accepted opinion in VLBI analysis has been to always estimate the gradients for every session. Among the a priori gradients, GRAD-2 performs best, independent from the number of observations. When having fewer than 3000 observations per session, GRAD-2 improves $44 \%$ of the BLRs by more than $1 \mathrm{~mm}$ while it degrades only $4 \%$ by more than $1 \mathrm{~mm}$ with respect to no a priori gradients. On the other hand, when having more than 3000 observations per session, GRAD-2 improves $41 \%$ of the BLRs by more than $1 \mathrm{~mm}$ while it degrades only $9 \%$ by more than 1 $\mathrm{mm}$ with respect to no a priori gradients. Also the empirical gradient model yields thorough results, particularly for a lower number of observations. When having fewer than 3000 observations per session, GPT3 $\left(1^{\circ} \times 1^{\circ}\right)$ improves $17 \%$ of the BLRs by more than $1 \mathrm{~mm}$ while it degrades only $3 \%$ by more than $1 \mathrm{~mm}$ with respect to no a priori gradients. On the other hand, when having more than 3000 observations per session, GPT3 $\left(1^{\circ} \times 1^{\circ}\right)$ improves $11 \%$ of the BLRs by more than $1 \mathrm{~mm}$ while it degrades $7 \%$ by more than $1 \mathrm{~mm}$ with respect to no a priori gradients. A further pleasant outcome of Tables 4 and 5 is that the results from GRAD- 2 are as good as those from the ray-traced delays, indicating that the approximation of the ray-traced delays using the extended gradient formula Eq. (5) works properly. The boundary of 3000 observations might appear a little general, as it does not consider the number of stations participating in a session or any geometry in the station constellation; however, it turned out to be very appropriate and useful. Alternatively, it would also be possible to apply tight absolute constraints to the gradients for sessions with a low number of observations instead of the a priori gradients. This, however, was not tested in this investigation.
The following itemization sums up all facts concerning the BLR analysis.

- The ray-traced delays, which represent the absolute reference values in this comparison, can be approximated perfectly well by using VMF1 plus the gradients GRAD1 as well as GRAD-2. In other words, this means that better BLRs can only be attained as soon as the ray-traced delays themselves become more accurate.

- Unlike the commonly accepted opinion, gradients shall not always be estimated within VLBI analysis. The design matrix in the least-squares adjustment must be sufficiently over-determined in order to produce reliable results. A certain criterion has to be fulfilled to ensure this, where the minimum value of 3000 observations per session turned out to be an approximate, but reliable boundary. Below this number, no gradients shall be estimated in VLBI analysis.

- Best results are achieved with the a priori gradients GRAD-2. However, GRAD-1 is only marginally worse but does not require a new gradient formula.

- Empirical a priori gradients generally have a considerably smaller effect on the resulting BLRs than discrete a priori gradients. In case no discrete a priori gradients are available, empirical gradients are most useful for VLBI sessions with few observations, where its usage yields much better BLRs than estimating the gradients in the analysis. GPT3 is marginally better that APG and DAO, whereas the difference between GPT3 $\left(5^{\circ} \times 5^{\circ}\right)$ and GPT3 $\left(1^{\circ} \times 1^{\circ}\right)$ is even more marginal.

- The topography has a significant influence on the resulting gradients, e.g., the presence of mountain ranges causes variant gradient values. For this reason, the provision on a grid with a coarse mesh size of $5^{\circ}$ seems to be insufficient, as the grid points are up to $550 \mathrm{~km}$ away from each other that makes it impossible to consider any topography in between. The mesh size of $1^{\circ}$ comes closer 


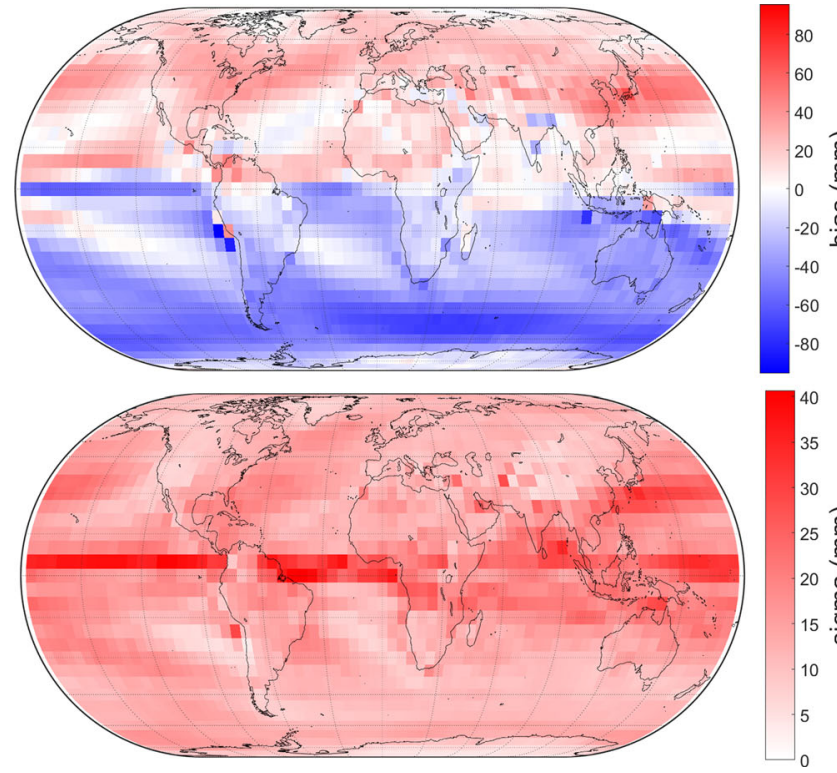

Fig. 8 Bias (top) and standard deviation (bottom) of the residuals in slant total delay at $5^{\circ}$ elevation and $180^{\circ}$ azimuth. Top left: bias of residuals between ray-tracing and disregarding azimuthal symmetry. Due to the atmospheric bulge, the residuals are generally positive in the northern hemisphere and generally negative in the southern hemi-

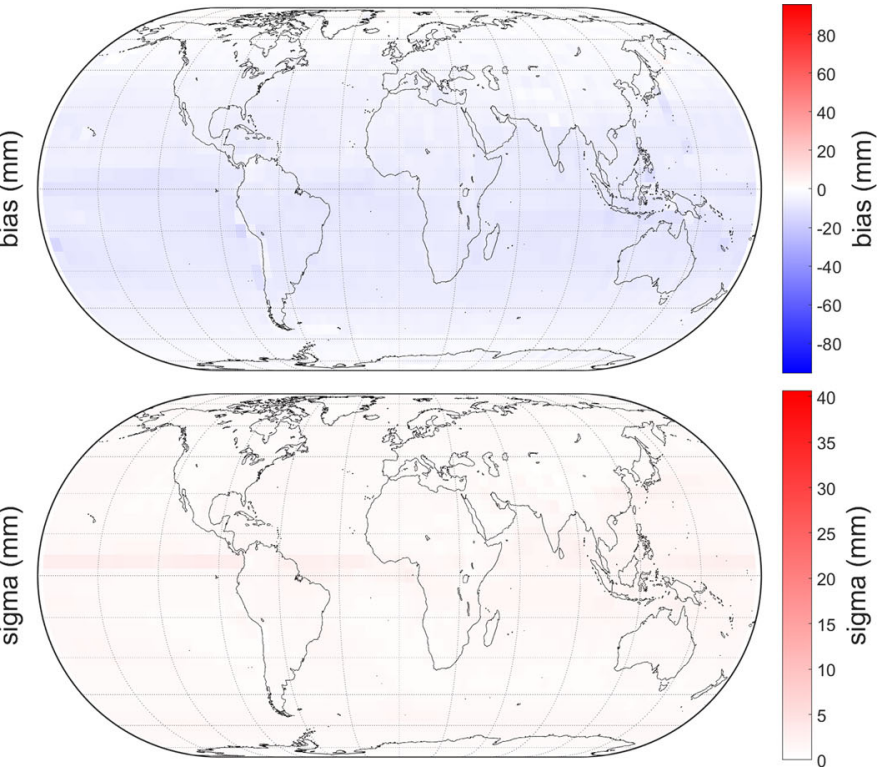

sphere. Top right: bias of residuals between ray-tracing and GRAD-1; the residuals are considerably lowered, albeit slightly negative. Bottom left and bottom right: the respective standard deviations. The application of GRAD-1 tremendously reduces the residuals at all levels as well

azimuths and 4 elevation angles. GRAD gradients are progressively applied in order to reveal their performance in reducing the residuals between the modeled delays and the ray-traced delays. Figures 8 and 9 feature the improvement of the residuals in bias and standard deviation, respectively, resulting from the application of GRAD.

Comparing mean absolute residuals or mean absolute error (MAE) is very meaningful, too. It describes the total difference to the reference values averaged over all observations, whereas the bias is always dependent on the algebraic sign. Table 6 lists mean absolute residuals for the different GRAD gradients averaged over all grid points and epochs, sorted by azimuth. The Vienna Mapping Functions 3 (VMF3) (Landskron and Böhm 2017) is used for modeling the azimuthally symmetric part of the delay. It does not matter here which GPT3 version to use, as the comparison is done for the $5^{\circ} \times 5^{\circ}$ grid intersection points.

From Table 6 the following conclusions can be drawn: global grid containing 2592 grid points. Ray-traced delays were generated for each grid point according to the specifications listed in Table 2. The ray-traced delays, regarded as the "true" values, are then compared to delays modeled with the three gradient formulae Eqs. (2), (5) and (6). The gradients LHG as well as DAO cannot be considered here, as they are only available for VLBI station locations and not for arbitrary points such as grid intersections.

This comparison is made concerning the residuals between the azimuth-wise ray-traced delays and those averaged over all azimuths for each of the 2592 grid points, 120 epochs, 8
- Due to the presence of an atmospheric bulge, azimuthal asymmetry is most pronounced in north and south direction and is least pronounced in east and west direction.

- The consideration of azimuthal asymmetry is of particular importance especially for small elevation angles like $5^{\circ}$.

- With the standard gradient formula of Chen and Herring (1997) (= GRAD-1), an improvement in the slant total 


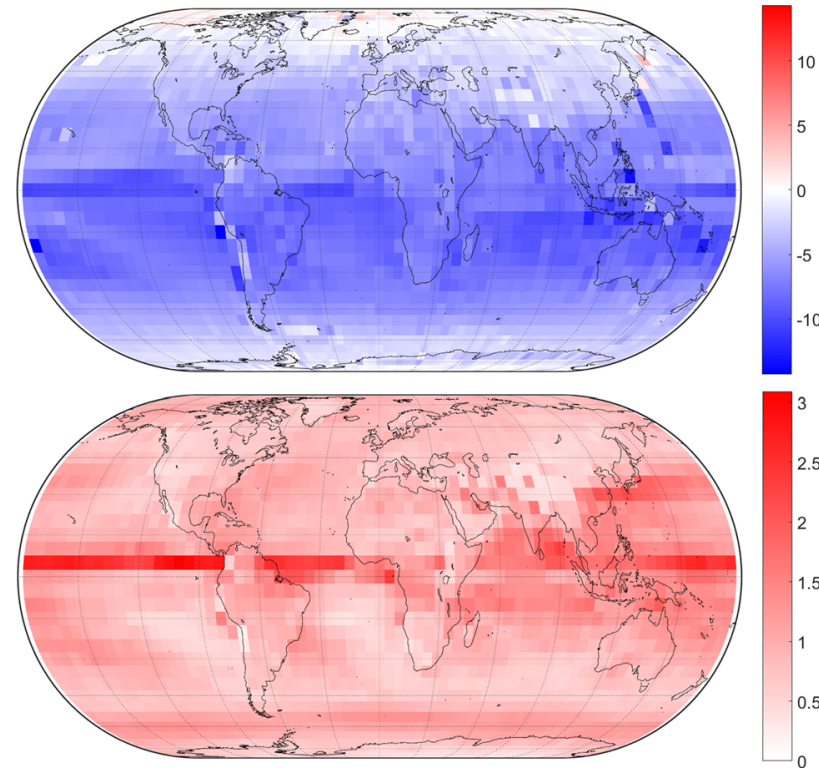

Fig. 9 Bias (top) and standard deviation (bottom) of the residuals in slant total delay at $5^{\circ}$ elevation and $180^{\circ}$ azimuth. Top left: bias of residuals between ray-tracing and applying GRAD-1; this is equal to the top right plot of Fig. 8, but differently scaled. Top right: bias of

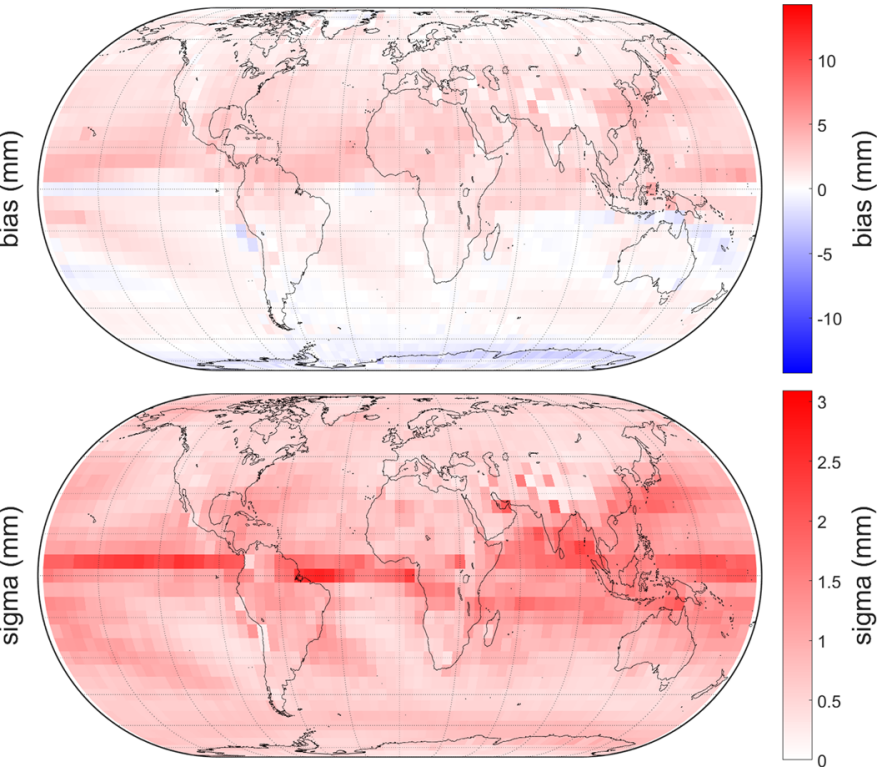

residuals between ray-tracing and GRAD-2. Thus, the negative residuals are mainly removed. Bottom left and bottom right: the respective standard deviations, showing no noticeable difference between them
Table 6 Mean absolute residuals $(\mathrm{mm})$ in slant total delay between ray-tracing and applying no gradient formula, the three GRAD gradients and empirical gradients, each for $5^{\circ}$ elevation and different azimuths $\alpha$, averaged over all 2592 grid points and 120 epochs from January 2001 to December 2010

\begin{tabular}{lcccccc}
\hline Gradient model & \multicolumn{7}{l}{ Mean abs. diff. in $\Delta L(\mathrm{~cm})$} \\
\cline { 2 - 6 } & $\alpha=0^{\circ}$ & $\alpha=45^{\circ}$ & $\alpha=90^{\circ}$ & $\alpha=135^{\circ}$ & $\alpha=180^{\circ}$ & Mean $\alpha$ \\
\hline No a priori gradients & 25.6 & 19.6 & 9.7 & 19.0 & 26.0 & 20.0 \\
GRAD-1 & 4.1 & 1.1 & 4.1 & 1.1 & 4.2 & 2.9 \\
GRAD-2 & 1.4 & 0.8 & 1.1 & 0.8 & 1.3 & 1.1 \\
GRAD-3 & 1.4 & 0.8 & 1.1 & 0.8 & 1.3 & 1.1 \\
APG & 16.4 & 14.4 & 10.8 & 13.0 & 16.8 & 14.3 \\
GPT3 & 9.4 & 7.5 & 7.4 & 7.5 & 9.5 & 8.3 \\
\hline
\end{tabular}

delays of up to $20 \mathrm{~mm}$ can be reached at $5^{\circ}$ elevation. On average, it improves the residuals by $86 \%$.

- Using the second gradient formula (=GRAD-2) further improves the slant total delays, although to a smaller degree. On average, the residuals are lower by notable 95\% compared to not considering azimuthal asymmetry.

- The third gradient formula (= GRAD-3) is not meaningful as it is not capable of further reducing the residuals compared to GRAD-2. This is most likely owing to insufficient over-determination in the least-squares adjustment, where six gradient variables shall be estimated from eight azimuths.

- The residuals when using empirical gradients are far off those from discrete gradients. However, GPT3 considerably improves the delays with respect to APG.

\section{Conclusions}

On the basis of ray-traced delays through numerical weather models (NWMs) using the highly sophisticated VieVS raytracer (Hofmeister and Böhm 2017), we developed new discrete horizontal gradients for a priori use in VLBI analysis referred to as GRAD, as well as a new empirical gradient model GPT3 in the two grid sizes $1^{\circ} \times 1^{\circ}$ and $5^{\circ} \times 5^{\circ}$. All of these models are capable of outperforming existing models in our comparisons; this is shown through baseline length repeatabilities (BLRs) from VLBI analyses as well as theoretical delays. An extended gradient formula including higher-order terms (GRAD-2) is able to simulate the raytraced delays with even higher precision than the standard gradient formula by Chen and Herring (1997). We found that the common estimation of gradients in VLBI analysis shall only be carried out under certain conditions. If the respective VLBI session exhibits fewer than 3000 observations, 
the gradient estimation rather degrades than improves the results. The sole usage of a priori gradients GRAD without additional gradient estimation is to be preferable in $90 \%$ of the VLBI sessions. However, as in general only a comparably small improvement can be achieved with the new models, we are forced to the conclusion that a big leap in the accuracy may only be achieved when the ray-traced delays and NWMs themselves become more accurate. This is supported by the fact that the ray-traced delays can be approximated already very well through the modeled gradients in all comparisons.

\section{Data and code availability}

Text files containing GRAD gradients can be downloaded from http://ggosatm.hg.tuwien.ac.at/DELAY/ETC/GRAD/. Information on the usage of the files is found in http:// ggosatm.hg.tuwien.ac.at/DELAY/readme.txt.

Acknowledgements Open access funding provided by Austrian Science Fund (FWF). The authors would like to thank the Austrian Science Fund (FWF) for financial support of this study within the projects RADIATE VLBI (P25320) and RADIATE ORD (ORD 86).

Open Access This article is distributed under the terms of the Creative Commons Attribution 4.0 International License (http://creativecomm ons.org/licenses/by/4.0/), which permits unrestricted use, distribution, and reproduction in any medium, provided you give appropriate credit to the original author(s) and the source, provide a link to the Creative Commons license, and indicate if changes were made.

\section{References}

Böhm J, Schuh H (2004) Vienna mapping functions in VLBI analyses. Geophys Res Lett 31:L01603. https://doi.org/10.1029/ 2003GL018984

Böhm J, Schuh H (2007) Troposphere gradients from the ECMWF in VLBI analysis. J Geod 81:403-408. https://doi.org/10.1007/ s00190-007-0144-2

Böhm J, Werl B, Schuh H (2006) Troposphere mapping functions for GPS and VLBI from European Centre for Medium-Range Weather Forecasts operational analysis data. J Geophys Res 111:B02406. https://doi.org/10.1029/2005JB003629

Böhm J, Spicakova H, Urquhart L, Steigenberger P, Schuh H (2011) Impact of a priori gradients on VLBI-derived terrestrial reference frames. In: Alef W, Bernhart S, Nothnagel A (eds) Proceedings of the 20th meeting of the European VLBI group for geodesy and astrometry. Schriftenreihe des Instituts für Geodäsie und Geoinformation der Universität Bonn, No. 22, pp 128-132, ISSN 1864-1113

Böhm J, Urquhart L, Steigenberger P, Heinkelmann R, Nafisi V, Schuh $\mathrm{H}$ (2013) A priori gradients in the analysis of space geodetic observations. In: Collileux X, Altamimi Z (eds) Proceedings of the IAG commission 1 symposium 2010 (REFAG2010), Paris, France, 48 October 2010, International Association of Geodesy Symposia Series

Böhm J, Möller G, Schindelegger M, Pain G, Weber R (2015) Development of an improved blind model for slant delays in the troposphere (GPT2w). GPS Solut 19:433. https://doi.org/10.1007/ s10291-014-0403-7

Böhm J, Böhm S, Boisits J, Girdiuk A, Gruber J, Hellerschmied A, Krasna H, Landskron D, Madzak M, Mayer D, McCallum J, McCallum L, Schartner M, Teke K (2017) Vienna VLBI and Satellite Software (VieVS) for Geodesy and Astrometry. Publications of the Astronomical Society of the Pacific, Accepted 2018

Chen G, Herring TA (1997) Effects of atmospheric azimuthal asymmetry on the analysis of space geodetic data. J Geophys Res 102(B9):20489-20502. https://doi.org/10.1029/97JB01739

Gardner CS (1977) Effects of horizontal refractivity gradients on the accuracy of laser ranging to satellites. Radio Sci 11(12):10371044. https://doi.org/10.1029/RS011i012p01037

Geerts B, Linacre E (1997) The height of the tropopause. University of Wyoming, Atmospheric Science, Laramie

Heinkelmann R, Tesmer V (2013) Systematic inconsistencies between VLBI CRF and TRF solutions caused by different analysis options. In: IAG Symposium, vol 138, Springer, Berlin. https://doi.org/10. 1007/978-3-642-32998-2_27

Herring TA (1992) Modeling atmospheric delays in the analysis of space geodetic data. In: Publications on Geodesy. In: DeMunck JC, Th. Spoelstra TA (eds) Proceedings of refraction of transatmospheric signals in Geodesy, vol 36. Netherlands Geodetic Commission Publications in Geodesy, The Hague, pp 157-164

Hobiger T, Ichikawa R, Koyama Y, Kondo T (2008) Fast and accurate ray-tracing algorithms for real-time space geodetic applications using numerical weather models. J Geophys Res 113:D20302. https://doi.org/10.1029/2008JD010503

Hofmeister A, Böhm J (2017) Application of ray-traced tropospheric slant delays to geodetic VLBI analysis. J Geod 91:945. https://doi. org/10.1007/s00190-017-1000-7

Jones FE (1981) The refractivity of Air. J Res Nat Bur Stand 86(1):27

Lagler K, Schindelegger M, Böhm J, Krasna H, Nilsson T (2013) GPT2: empirical slant delay model for radio space geodetic techniques. Geophys Res Lett 40(6):1069-1073. https://doi.org/10.1002/grl. 50288

Landskron D (2017) Modeling tropospheric delays for space geodetic techniques. Dissertation, Department of Geodesy and Geoinformation, TU Wien, Supervisor: J. Böhm. http://repositum.tuwien. ac.at/urn:nbn:at:at-ubtuw:1-100249

Landskron D, Böhm J (2017) VMF3/GPT3: refined discrete and empirical troposphere mapping functions. J Geod. https://doi.org/10. 1007/s00190-017-1066-2

Landskron D, Möller G, Hofmeister A, Böhm J, Weber R (2015a), Refined and site-augmented tropospheric delay models in the analysis of VLBI observations. In: 26th IUGG general assembly, Prague, 2015/06/22-2007/07/02

Landskron D, Hofmeister A, Böhm J (2015b), Refined tropospheric delay models for CONT11. In: International association of Geodesy symposia. Springer, Berlin, pp 1-5. https://doi.org/10. 1007/1345_2015_56

Landskron D, Hofmeister A, Mayer D, Böhm J (2016), Six hourly time series of horizontal troposphere gradients in VLBI analysis. In: European geosciences union general assembly 2016, Vienna, 2016/04/17-2016/04/22

Lyard F, Lefevre F, Letellier T, Francis O (2006) Modelling the global ocean tides: modern insights from FES2004. Ocean Dyn 56:394415

MacMillan DS, Ma C (1997) Atmospheric gradients and the VLBI terrestrial and celestial reference frames. Geophys Res Lett 24(4):453-456. https://doi.org/10.1029/97GL00143

Mayer D, Böhm J, Krasna H, Landskron D (2017) Tropospheric delay modelling and the celestial reference frame at radio wavelengths. Astron Astrophys. https://doi.org/10.1051/0004-6361/ 201731681 
Nilsson T, Böhm J, Wijaya DD, Tresch A, Nafisi V, Schuh H (2013) Path delays in the neutral atmosphere. In: Böhm J, Schuh H (eds) Atmospheric Effects in Space Geodesy. Springer, Berlin ISBN: 978-3-642-36931-5

Spicakova H, Plank L, Nilsson T, Böhm J, Schuh H (2011) Terrestrial reference frame solution with the Vienna VLBI Software VieVS and implication of tropospheric gradient estimation. In: Proceedings of the 20th meeting of the European VLBI group for Geodesy and sstrometry (EVGA)
Titov O (2009) A new estimator for VLBI baseline length repeatability. J Geod 83:1041-1049. https://doi.org/10.1007/s00190-009-0322-5

Wijaya DD, Böhm J, Karbon M, Krasna H, Schuh H (2013) Atmospheric pressure loading. Chapter 4 in atmospheric effects in space geodesy. In: Böhm J, Schuh H (eds) Springer, Berlin. ISBN: 9783642369322 (online) 